\title{
Composição química de íons majoritários na deposição úmida da cidade de São José do Rio Preto, Brasil
}

The chemical composition of major ions in wet deposition from São José do Rio Preto city, Brasil

Composición química de iones majoritarios en la deposición úmida de la ciudad de São José do Rio Preto, Brasil

Fabio Henrique Medeiros Mestre, UNESP, Brasil fabiohmed@gmail.com

Flavia Carla dos Santos Martins Mestre, UNESP, Brasil flaviacarlamartins@hotmail.com

Márcia Cristina Bisinoti Professor Livre-Docente, UNESP, Brasil. mbisinoti@hotmai.com 


\section{RESUMO}

Este trabalho tem como principal objetivo avaliar a influência da produção de álcool da cana-de-açúcar e da atividade industrial em uma cidade do estado de São Paulo na ciclagem de íons majoritários. Foram coletadas 103 amostras de deposição total e 96 amostras de deposição úmida apenas após cada evento de chuva usando três coletores na região noroeste do estado de São Paulo, entre março de 2009 e maio de 2010. Os parâmetros $\mathrm{NH}_{4}^{+}, \mathrm{NO}_{3}{ }^{-}, \mathrm{SO}_{4}{ }^{2-}, \mathrm{PO}_{4}{ }^{3-}, \mathrm{K}^{+}, \mathrm{pH}^{2}$ condutividade foram quantificados. Os resultados mostraram que as maiores variações nas amostras de deposição úmida ocorreu na seguinte ordem; $\mathrm{PO}_{4}{ }^{3-}>\mathrm{K}^{+}>\mathrm{NO}_{3}{ }^{-}>\mathrm{NPOC}>\mathrm{NH}_{4}{ }^{+}>\mathrm{C}$. E. $>\mathrm{SO}_{4}{ }^{2-} \sim \mathrm{TOC}>>\mathrm{pH}$. Os maiores valores de $\mathrm{pH}$ foram observados durante o período de colheita $(4,8$ e 7,3$)$. As concentrações de média ponderada do volume (VWM) para a deposição total e somente para molhado foram maiores durante o período de queima de cana para $\mathrm{K}^{+}, \mathrm{NH}_{4}{ }^{+}$, $\mathrm{SO}_{4}{ }^{2-}, \mathrm{PO}_{4}{ }^{3-}$ and $\mathrm{NO}_{3}$. Para TOC e NPOC, maior VWM foram observados durante o período de queima de cana-de-açúcar para amostras de deposição total, observando-se o comportamento oposto para a deposição úmida. Para os valores de $\mathrm{K}^{+}, \mathrm{NH}_{4}{ }^{+}$e $\mathrm{SO}_{4}{ }^{2-} \mathrm{VWM}$ obtidos foram até 3,5 vezes maiores do que os valores encontrados para outras cidades impactadas pelas atividades de cana-de-açúcar no Estado de São Paulo. Com base nesses resultados, podemos ver que, durante o período de colheita de cana-de-açúcar, houve aumento em $\mathrm{K}^{+}, \mathrm{NH}_{4}{ }^{+}, \mathrm{PO}_{4}{ }^{3-}$ e $\mathrm{NO}_{3}$, indicando que esta prática contribui para a ciclagem desses elementos.

PALAVRAS-CHAVE: ions, deposição total, São José do Rio Preto.

\section{ABSTRACT}

This work concerns the characteristics and the influence of sugarcane alcohol production and industrial activity in a city in the state of São Paulo on the cycling of major ions. A total of 103 samples of total deposition and 96 samples of wetonly deposition were sampled after each rain event using three collectors in the northwest area of São Paulo State, Brazil between March 2009 and May 2010. The parameters $\mathrm{NH}_{4}{ }^{+}, \mathrm{NO}_{3}{ }^{-}, \mathrm{SO}_{4}{ }^{2-}, \mathrm{PO}_{4}{ }^{--}, \mathrm{K}+, \mathrm{pH}$ and conductivity were quantified. Results showed that the highest variations in wet-only deposition samples occurred in the following order; $\mathrm{PO}_{4}{ }^{3-}>\mathrm{K}^{+}>\mathrm{NO}_{3}{ }^{-}>\mathrm{NPOC}>\mathrm{NH}_{4}{ }^{+}>\mathrm{C}$.E. $>\mathrm{SO}_{4}{ }^{2-} \sim \mathrm{TOC}>>\mathrm{pH}$. The highest $\mathrm{pH}$ values were observed during the harvest period (4.8 and 7.3). The volume-weighted mean (VWM) concentrations for total and wet-only deposition were higher during the period of sugarcane burning for $\mathrm{K}^{+}, \mathrm{NH}_{4}{ }^{+}, \mathrm{SO}_{4}{ }^{2-}, \mathrm{PO}_{4}{ }^{3-}$ and $\mathrm{NO}_{3}{ }^{-}$. For TOC and NPOC higher VWM were observed during the sugarcane burning period for total deposition samples, the opposite behavior being observed for wet-only deposition. For $\mathrm{K}^{+}, \mathrm{NH}_{4}{ }^{+}$and $\mathrm{SO}_{4}{ }^{2-} \mathrm{VWM}$ values obtained were up to 3.5 times higher than the values found for other cities impacted by sugarcane activities in São Paulo State. Based on these results we can see that during the period of harvesting sugarcane there was an increase in $\mathrm{K}^{+}, \mathrm{NH}_{4}^{+}, \mathrm{PO}_{4}{ }^{3-}$ and $\mathrm{NO}_{3}{ }^{-}$, indicating that this practice contributes to the cycling of these elements.

KEY-WORDS: ions, total deposition, São José do Rio Preto.

\section{RESUMO}

Este trabajo se refiere a las características ya la influencia de la producción de alcohol de la cana-de-azúcar y de la actividad industrial en una ciudad del estado de São Paulo en la ciclagem de grandes íons. Foram coletadas 103 muestras de deposición total y 96 muestras de deposición de úmida sólo después de cada evento de lluvia utilizando tres colectores en la región noroeste del estado de São Paulo, entre marzo de 2009 y mayo de 2010. Os desenho $\mathrm{NH}_{4}{ }^{+}, \mathrm{NO}_{3}{ }^{-}$, $\mathrm{SO}_{4}{ }^{2-}, \mathrm{PO}_{4}{ }^{3-}, \mathrm{K}^{+}, \mathrm{pH}$ y conductividad foram cuantificados. Los resultados muestran que los mayoras variaciones en las muestras de deposición sólo para molar ocorre en la siguiente orden; $\mathrm{PO}_{4}{ }^{3-}>\mathrm{K}^{+}>\mathrm{NO}_{3}^{-}>\mathrm{NPOC}^{-} \mathrm{NH}_{4}^{+}>\mathrm{C}$.E. $>\mathrm{SO}_{4}{ }^{2-} \sim$ TOC > pH. Los mayores valores de $\mathrm{pH}$ fueron observados durante el período de colheita $(4,8$ y 7,3$)$. Como concentraciones de media ponderada do volume (VWM) para un total de depósitos para el período de queima de cana para $\mathrm{K}^{+}, \mathrm{NH}_{4}{ }^{+}, \mathrm{SO}_{4}{ }^{2-}, \mathrm{PO}_{4}{ }^{3-}$ y $\mathrm{NO}_{3}$. Para TOC y NPOC, mayor VWM fueron observados durante el período de queima de la cana-de-azúcar para muestras de la deposición total, observando-se que el comportamiento para una deposición de humedad sólo. Para los valores de $\mathrm{K}^{+}, \mathrm{NH}_{4}{ }^{+}$y $\mathrm{SO}_{4}{ }^{2} \mathrm{VWM}$ obtidos para hasta 3,5 veces mayores que los valores encontrados para otras ciudades impactadas por actividades de cana-de-azúcar no Estado de São Paulo. Com base nesses resultados, podemos ver que, durante el período de colheita de cana-de-azúcar, hubo un aumento en $\mathrm{K}^{+}, \mathrm{NH}_{4}{ }^{+}$, $\mathrm{PO}_{4}{ }^{3-}$ y $\mathrm{NO}_{3}{ }^{-}$, indicando que esta práctica contribuye para el ciclismo de elementos.

PALAVRAS-CHAVE: iones, total deposición, São José do Rio Preto. 


\section{Introdução}

O principal mecanismo de transporte de poluentes é a deposição úmida, onde há remoção de partículas e gases da atmosfera devido à precipitação, podendo afetar ecossistemas como rios, lagos e solos. O outro mecanismo, chamado deposição seca ocorre geralmente pela adsorção de poluentes na fração grossa do particulado, devido à ação da gravidade sobre as partículas (CASTANHO, 1999; SOUZA et al., 2006).

Os principais poluentes transportados por estes mecanismos incluem compostos de nitrogênio, enxofre e carbono geralmente associados com o material particulado na atmosfera. A queima incompleta de combustíveis fósseis, as atividades industriais e as diferentes fontes de energia bem como a agricultura têm contribuído para as emissões de poluentes como $\mathrm{CO}_{2}, \mathrm{NH}_{3}, \mathrm{H}_{2} \mathrm{SO}_{4}$, enxofre, óxidos de nitrogênio e óxidos de metal. Estes compostos químicos, uma vez na atmosfera, podem dissolver-se na precipitação e, em seguida, incorporar-se rapidamente nos ciclos fundamentais produzindo mudanças permanentes nos processos geoquímicos dos ecossistemas afetados (MIGLIAVACCA, 2009).

Devido à presença natural de dióxido de carbono atmosférico dissolvido, o valor do pH da água de chuva não-poluída é de cerca de 5,6. Contudo, a formação de poluentes secundários, tais como $\mathrm{H}_{2} \mathrm{SO}_{4}$ e $\mathrm{HNO}_{3}$, ambos muito solúveis e ácidos fortes, pode acarretar no aumento da acidez, provocando a chuva ácida, a qual vem sendo bastante estudada em razão de prejuízos ecológicos e econômicos que ocasiona. Vale ressaltar que, considerando o processo de formação destes ácidos inorgânicos, verifica-se que a chuva ácida pode ser gerada em locais bem distantes das fontes poluidoras (CARVALHO JUNIOR, 2004; BAIRD, 2002; MARTINS E ANDRADE, 2002).

Estudos mostraram que as reações de neutralização junto à deposição seca e úmida parecem ser os meios mais comuns de remoção dos compostos ácidos da atmosfera, uma vez que parte dos ácidos sulfúrico e nítrico no ar, em contato com amônia gasosa, é neutralizada formando sais de amônio na forma de material particulado. Embora estes sais sejam formados inicialmente em partículas aquosas, a evaporação da água pode resultar na formação de partículas sólidas. Os íons predominantes nas partículas finas são os ânions sulfato $\mathrm{SO}_{4}{ }^{2-}$, bissulfato $\mathrm{HSO}^{4-}$ e nitrato $\mathrm{NO}^{3-}$, e os cátions $\mathrm{NH}^{4+}$ e o íon hidrogênio $\mathrm{H}^{+}$(FELIX E CARDOSO, 2004; BAIRD, 2002). Destaca-se também que, sendo a deposição atmosférica um dos principais mecanismos de ciclagem e redistribuição dos vários elementos químicos sobre a superfície do planeta, o conhecimento quantitativo e 
qualitativo das mesmas é de grande importância para o entendimento dos ciclos biogeoquímicos de elementos e da influência das atividades antrópicas nestes processos (PIRES, 2005; SOUZA et al., 2006; SILVA, 2006).

A cidade de São José do Rio Preto (SJRP), localizada no Noroeste do Estado de São Paulo (Figura 1) possui clima tropical de altitude, definido por meses secos (Abril - Novembro) e úmidos (Dezembro - Março), com esses períodos coincidindo com a temporada de safra e entressafra da cana-deaçúcar, respectivamente. Com 410.000 habitantes, a cidade de SJRP tem como sua principal atividade econômica a agricultura, com destaque a cultura canavieira. Além disso, SJRP possui uma frota de aproximadamente 200.000 veículos (DER, 2006), bem como de rodovias de importância nacional, como a Washington Luís (SP-310) e a Br-153. Outro ponto a destacar é que o estado de São Paulo é responsável por $60 \%$ da produção brasileira de açúcar e álcool, e a região de São José do Rio Preto já conta com 12\% deste total produzidos por 15 usinas (ANDRADE, 2009; CONJUNTURA ECONÔMICA, 2010; MAPA/SPAE, 2007).

Frente ao exposto, o objetivo do presente estudo foi caracterizar a composição química da água de chuva (ou precipitação atmosférica) na cidade de São José do Rio Preto identificando as fontes dos principais elementos majoritários.

\section{Materiais e Métodos}

\subsection{Condições de amostragem}

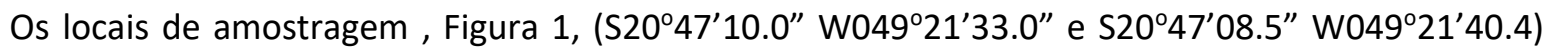
foram configuradas de acordo com a Agência Ambiental do Estado de São Paulo (CETESB), em uma preferência de direção de vento, em uma cobertura de área aberta Pelo menos $800 \mathrm{~m} 2$ e livre de fontes de emissão nas proximidades. A cidade de São José do Rio Preto fica a $400 \mathrm{~km}$ da cidade de São Paulo. A população é de cerca de 410 mil habitantes. As amostras de águas pluviais foram recolhidas, após cada evento de chuva, em triplicado, de março de 2009 a setembro de 2010, sendo 103 da deposição total (TD) e 96 da deposição de umidade úmida (WD). Todos os coletores são montados a 1,5 metros do chão. Para as amostras de deposição úmida apenas foram empregadas um coletor automático (Biotecnosis $\mathrm{CCH}$ 08) e para a deposição total foram empregados coletores manuais. 


\section{Periódica Eletrânica}

\section{Fórum Ambiental}

Figura 1. Mapa com a localização dos locais de amostragem.
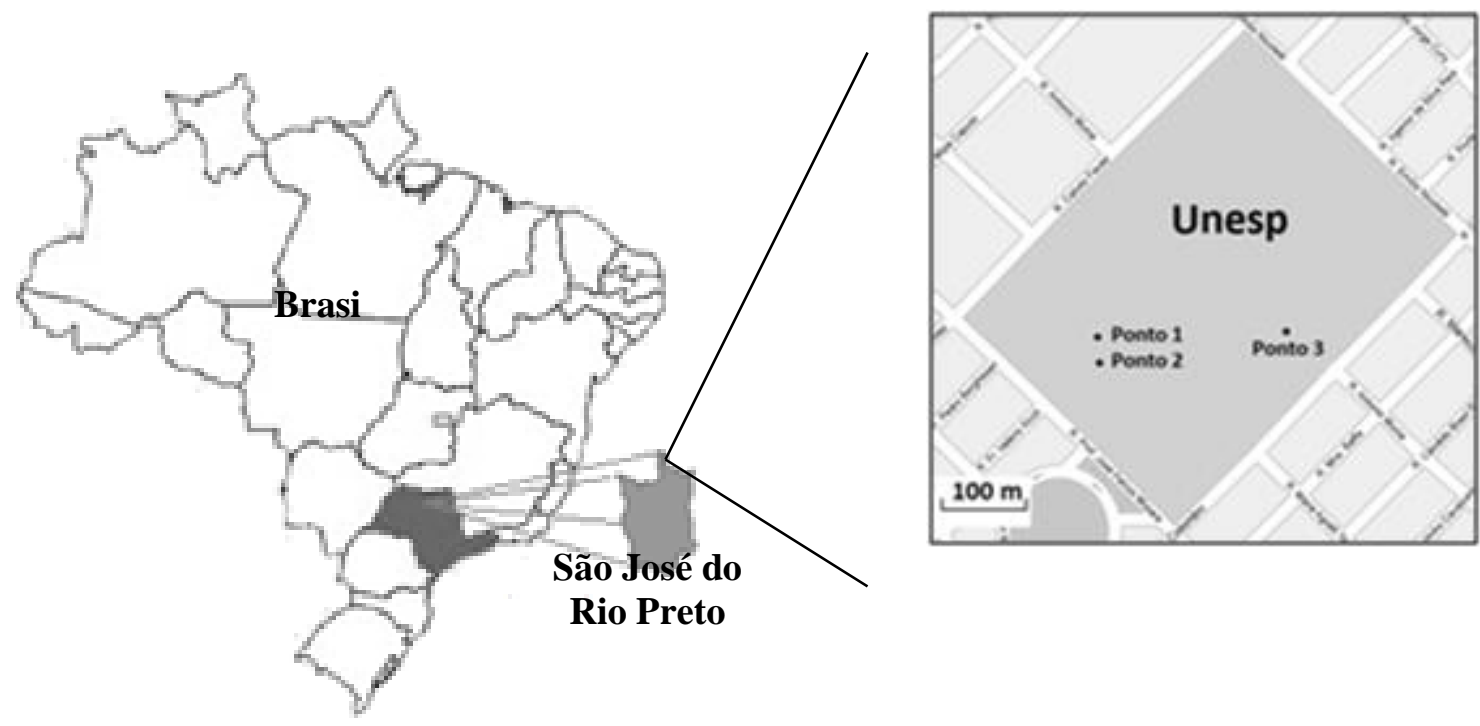

\subsection{Materiais e métodos}

Imediatamente após cada evento de chuva, os frascos coletores foram removidos do amostrador de água. Todas os frascos empregados para a estocagem das amostras foram em polietileno de alta densidade e foram mantidos em meio ácido e enxaguados várias vezes com água ultrapura anterior ao uso, com exceção do frasco empregado para a amostragem de Carbono Orgânico Total, no qual foi empregado frascos de vidro ambar. $\mathrm{pH}$ e condutividade foram analisados logo após a coleta em equipamentos previamente calibrados. Sulfato $\left(\mathrm{SO}_{4}{ }^{2-}\right)$ foi analisado pelo método turbidimétrico $\left(4500 \mathrm{SO}_{4}{ }^{2-} \mathrm{E}\right)$. Ânions majoritários como nitrato $\left(\mathrm{NO}_{3}{ }^{-}\right)$, fosfato $\left(\mathrm{PO}_{4}{ }^{3-}\right)$ e amônia $\left(\mathrm{NH}_{4}{ }^{+}\right)$foram quantificados empregando método espectrofotométricos (4500 $\mathrm{NO}_{3}^{-} \mathrm{B}, 4500 \mathrm{P}-\mathrm{E}$ e método do ácido salicílico) (Apha, 2005; Krom, 1980). Carbono Orgânico Total (TOC) e Carbono Orgânico Não-Purgáve foram determinados empregando Analisador de Carbono Orgânico Total (Shimadzu, TOC-VCSH). Potássio $\mathrm{K}^{+}$foi analisad por Espectrometro de Absorção Atômica com Atomização por Chama no modo emissão (Varian, AA240FS) e o método de adição de padrão foi usado para evitar a supressão de íon. Para todas as quantificações foram empregados água ultrapura (Millipore System, Milli-QPlus model). O laboratório onde estas amostras foram 
analisadas participa de Ensaio de Proficiência pelo INMETRO e EMBRAPA obtendo sempre resultados satisfatórios ( $z$ score menor que 2). As amostras foram analisadas o mais rápido possível e em conformidade com os métodos oficiais.

\subsection{Média Ponderada pelo Volume}

As Médias Ponderadas pelo Volume (VWM) foram calculados para cada evento de chuva, sendo divididos em três períodos: VWM geral, que inclui todo o período de amostragem, a atividade de cana-de-açúcar VWM (março a novembro) e entre safra VWM (dezembro a fevereiro). Para os anos de 2009/2010 foram coletadas 103 (68/35) amostras de deposição total, com 49 (44/5) amostras representativas do período de atividade da cana-de-açúcar e 54 (24/30) amostras para o período de entressafra. Para a deposição úmida apenas nos anos de 2009/2010 foram coletadas amostras 96 (62/34), sendo 48 (43/5) amostras do período de atividade da cana-de-açúcar e 48 (19/29) amostras de entressafra de cana-de-açúcar.

\section{Resultados e Discussão}

Os coeficientes médios, mínimos, máximos e de variação dos parâmetros medidos na precipitação da atmosfera da cidade de São José do Rio Preto estão listados na Tabela 1. As maiores variações foram observadas para $\mathrm{PO}_{4}^{3-}(662 \%)$ e $\mathrm{K}+(490 \%)$, seguido de variações menos importantes na seguinte ordem $\mathrm{NO}_{3}{ }^{-}>\mathrm{NPOC}>\mathrm{NH}_{4}{ }^{+}>\mathrm{C}$.E. $>\mathrm{SO}_{4}{ }^{2-} \sim \mathrm{TOC}>\mathrm{pH}$, sendo que para $\mathrm{K}^{+}$, $\mathrm{NH}_{4}{ }^{+}, \mathrm{SO}_{4}{ }^{2-}, \mathrm{PO}_{4}{ }^{3-}$ e $\mathrm{NO}_{3}{ }^{-}$, os valores variaram entre o limite de detecção, $\mathrm{LD}(0,0004,0,02,0,05$, 0,01 e $0,07 \mathrm{mg} \mathrm{L}^{-1}$ ) para 13,6,5,3, 14,8, 29,9 e 5,6 mg L-1, respectivamente. As concentrações de TOC e NPOC variaram de 0,41 a 13,9 e 0,25 a 21,5 mg L-1, respectivamente. Os valores mínimos e máximos observados para a condutividade foram de 2,1 e 72,4 $\mu \mathrm{S} \mathrm{cm-1} \mathrm{e} \mathrm{para} \mathrm{pH} \mathrm{4,8} \mathrm{e} \mathrm{7,3.}$ 
Tabela 1: Média, valores mínimos (Min.), Máximo (Máx.) e coeficiente de variação (CV) para deposição úmida apenas na cidade de São José do Rio Preto, de março de 2009 a setembro de 2010.

\begin{tabular}{|c|c|c|c|c|}
\hline Compostos & & Média & Mín. - Máx. & CV (\%) \\
\hline $\mathrm{K}^{+}$ & & 0,30 & $<0,0004-13,6$ & 490 \\
\hline $\mathrm{NH}_{4}^{+}$ & & 0,51 & $<0,02-5,3$ & 157 \\
\hline $\mathrm{SO}_{4}{ }^{2-}$ & 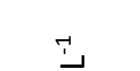 & 3,6 & $<0,05-14,8$ & 98 \\
\hline $\mathrm{PO}_{4}{ }^{3-}$ & $\stackrel{\infty}{\xi}$ & 0,6 & $<0,01-29,9$ & 662 \\
\hline $\mathrm{NO}_{3}{ }^{-}$ & & 0,40 & $<0,07-5,60$ & 178 \\
\hline TOC & & 1,9 & $0,41-13,9$ & 97 \\
\hline NPOC & & 2,1 & $0,25-21,5$ & 161 \\
\hline $\mathrm{pH}$ & - & 6,0 & $4,8-7,3$ & 9 \\
\hline C.E. & $\mu \mathrm{S} \mathrm{cm} \mathrm{cm}^{-2}$ & 8,4 & $2,10-72,4$ & 114 \\
\hline
\end{tabular}

A distribuição do pH em todas as amostras é mostrada na Figura 2. Cerca de $18 \%$ das amostras de chuva apresentaram um pH inferior a 5,6, característico da água de chuva ácida (CHARLSON; RODHE, 1982), sendo $76 \%$ dessas amostras coletadas durante o período de atividade da cana-deaçúcar. A maior fração (80\%) das amostras de águas pluviais teve um pH entre 5,6 e 7,0 e a fração das amostras com $\mathrm{pH}>7,0$ é de apenas $2 \%$. As hipóteses de $\mathrm{pH}$ superior a 5,6 observadas na cidade de São José do Rio Preto são devidas a cinzas incorporadas à atmosfera durante a cana-deaçúcar que apresentam uma natureza alcalina. 
Figura 2. Frequência distribuição do pH para deposição úmida

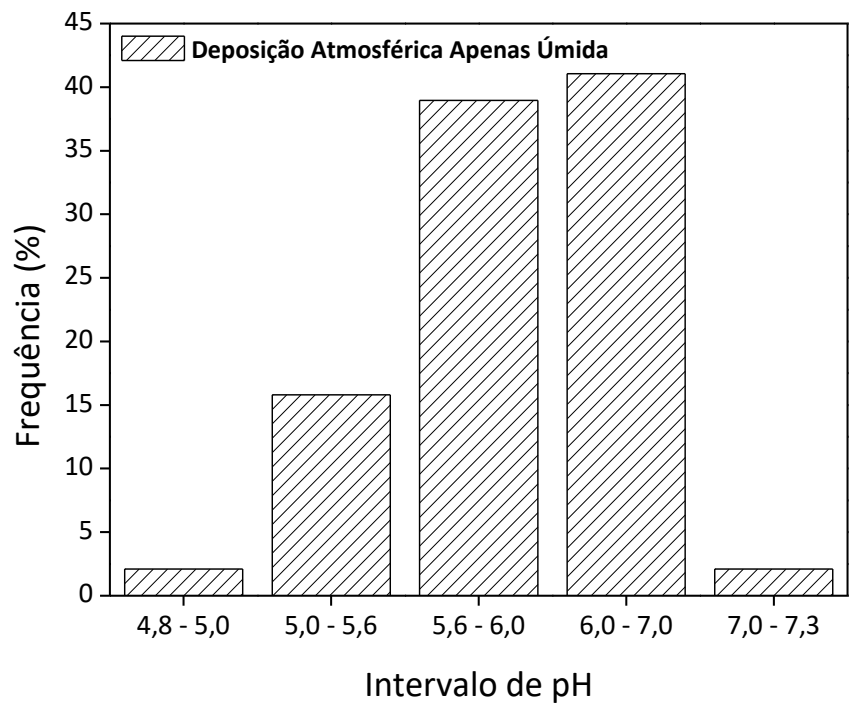

Os resultados apresentados na Figura 3 ilustram que o pH das amostras é maior nos meses após o foco de queimadas (INPE, 2011) e seguiu uma precipitação atmosférica acumulada, abaixo de 50 $\mathrm{mm}$. Isto é observado em junho de 2009, na medida em que o pH foi de 6,5 (durante os meses de abril e maio de 2009 foram registrados 27 e 28 focos de queimadura e precipitação de chuva acumulada de 7 e $3 \mathrm{~mm}$, respectivamente). A Figura 3 mostra que um aumento de pH após o mês de abril de 2010 (com cerca de 49 focos de queimadas e $2 \mathrm{~mm}$ de pluviosidade acumulada), o maior valor de $\mathrm{pH}(7,28)$ foi observado no mês de junho De 2010 , no qual foi observado que os meses anteriores com os maiores volumes de focos de queimada (195 e 197 respectivamente) na região em conjunto com menores pluviosidades mensais acumulada (cerca de $2 \mathrm{~mm}$ em cada mês), sugerindo com isso focos de queimadas influenciam um caráter básico na água da chuva da região de SJRP. 


\section{Periódica Eletrânica}

\section{Fórum Ambiental}

Volume 14, Número 1, 2018

da Alta Paulista

ISSN 1980-0827

Figura 3. Relação do $\mathrm{pH}$, focos de queimadas e precipitação media acumulada na região de SJRP durante o período de Março de 2009 a Junho de 2010.

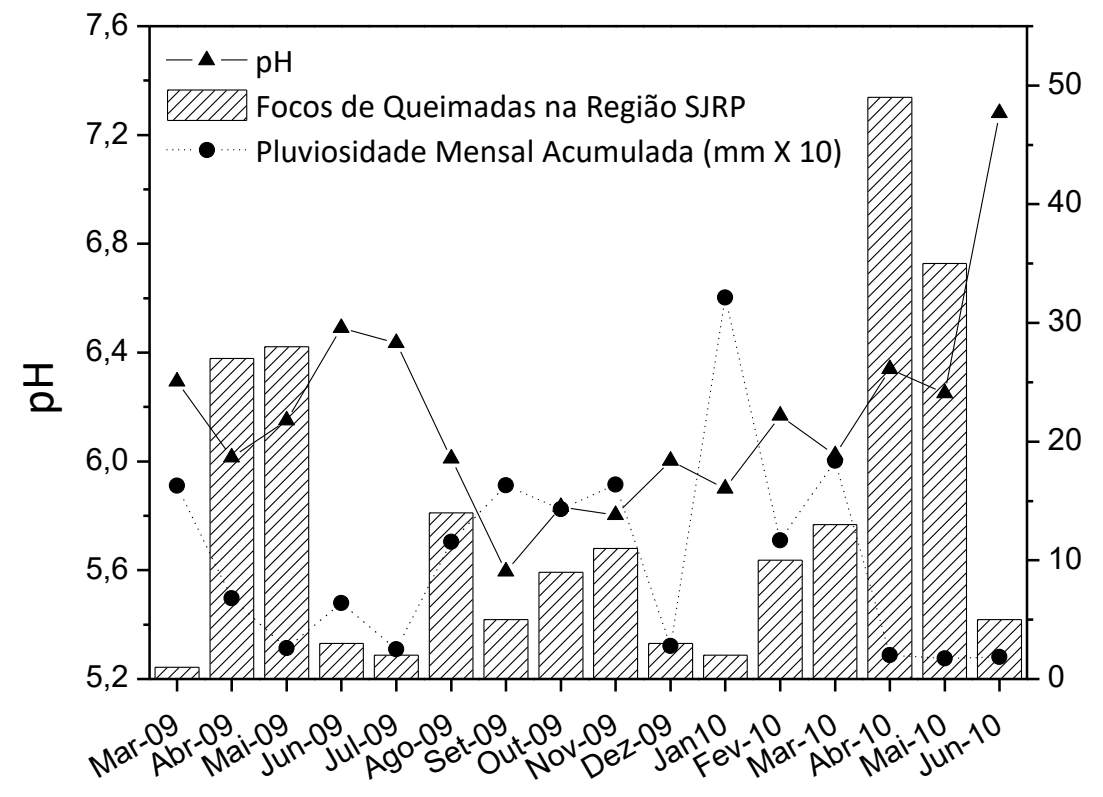

Foi observada uma baixa correlação $(0,34)$ entre pH e C.E., e correlações negativas entre pH e volume de chuva em cada evento $(r=-0,44)$ e para C.E. e volume de chuva $(r=-0,34)$.

\subsection{Média Ponderada Pelo Volume de Espécies Químicas Provenientes da Deposição Atmosférica}

Para as amostras de DT e DU (Tabela 2) foi observado que a maior MPV ocorreu no período de safra da cana-de-açúcar para os elementos $\mathrm{K}^{+}\left(0,68\right.$ e $\left.0,17 \mathrm{mg} \mathrm{L}^{-1}\right), \mathrm{NH}_{4}{ }^{+}\left(1,31\right.$ e $\left.0,38 \mathrm{mg} \mathrm{L}^{-1}\right), \mathrm{SO}_{4}{ }^{2-}$ $\left(5,99\right.$ e $\left.4,70 \mathrm{mg} \mathrm{L}^{-1}\right), \mathrm{PO}_{4}{ }^{3-}\left(3,08\right.$ e $\left.0,48 \mathrm{mg} \mathrm{L}^{-1}\right)$ e $\mathrm{NO}_{3}^{-}\left(0,94\right.$ e $\left.0,40 \mathrm{mg} \mathrm{L}^{-1}\right)$.

Já para TOC e NPOC (Tabela 2) foi observado que o maior valor de MPV ocorreu no período de safra da cana-de-açúcar para as amostras de DT (7,12 e 5,99 $\left.\mathrm{mg} \mathrm{L}^{-1}\right)$ e para as amostras de DU a maior MPV ocorreu no período de entressafra da cana-de-açúcar $\left(1,77\right.$ e 1,76 $\left.\mathrm{mg} \mathrm{L}^{-1}\right)$. 
Tabela 1: Média ponderada pelo volume (MPV) para as amostras de DU para o período de safra da cana-de-açúcar (Safra) e período de entressafra (Entressafra) da cana-de-açúcar e seus respectivos coeficientes de variação (CV) para a deposição atmosférica da cidade de SJRP.

\begin{tabular}{|c|c|c|c|c|c|c|c|c|}
\hline & \multicolumn{4}{|c|}{$\begin{array}{l}\text { Média Ponderada Pelo Volume das } \\
\text { Amostras de DT }\end{array}$} & \multicolumn{4}{|c|}{$\begin{array}{l}\text { Média Ponderada Pelo Volume } \\
\text { das Amostras de DU }\end{array}$} \\
\hline & Safra & CV & $\begin{array}{l}\text { Entres- } \\
\text { safra }\end{array}$ & CV & Safra & CV & $\begin{array}{l}\text { Entres- } \\
\text { safra }\end{array}$ & CV \\
\hline $\mathrm{K}^{+}$ & 0,68 & 22 & 0,20 & 10 & 0,17 & 19 & 0,05 & 20 \\
\hline $\mathrm{NH}_{4}^{+}$ & 1,31 & 31 & 0,54 & 15 & 0,38 & 19 & 0,33 & 19 \\
\hline $\mathrm{SO}_{4}{ }^{2-}$ & 5,99 & 16 & 2,20 & 5 & 4,70 & 18 & 1,72 & 20 \\
\hline $\mathrm{PO}_{4}^{3-}$ & 3,08 & 35 & 0,12 & 67 & 0,48 & 87 & 0,01 & 24 \\
\hline $\mathrm{NO}_{3}^{-}$ & 0,94 & 16 & 0,49 & 15 & 0,40 & 27 & 0,14 & 17 \\
\hline TOC & 7,12 & 26 & 5,36 & 10 & 1,35 & 15 & 1,77 & 21 \\
\hline NPOC & 5,99 & 25 & 5,15 & 10 & 1,20 & 14 & 1,76 & 20 \\
\hline
\end{tabular}

Para K $\mathrm{K}^{+}\left(0,68 \mathrm{mg} \mathrm{L}^{-1}\right), \mathrm{NH}_{4}{ }^{+}\left(1,3 \mathrm{mg} \mathrm{L}^{-1}\right)$ e $\mathrm{SO}_{4}{ }^{2-}\left(6,0 \mathrm{mg} \mathrm{L}^{-1}\right)$ os valores de MPV (Tabela 3) para a cidade de SJRP foram até 3,5 vezes superiores aos de cidades impactadas pelo cultivo da cana-deaçúcar no estado de SP como Ribeirão Preto $\left(0,04 ; 0,50\right.$ e 1,6 $\left.\mathrm{mg} \mathrm{L}^{-1}\right)$, Campinas $(0,05 ; 0,27$ e 1,0 $\left.\mathrm{mg} \mathrm{L}^{-1}\right)$ e Santa Maria da Serra $\left(0,14 ; 0,26\right.$ e 0,59 $\left.\mathrm{g} \mathrm{L} \mathrm{L}^{-1}\right)$ e a cidade de Figueira $(0,39 ; 0,54$ e 3,3 mg $\mathrm{L}^{-1}$ ), uma cidade que possui uma usina termoelétrica à base de carvão, no Paraná. Já o $\mathrm{NO}_{3}{ }^{-}$ apresentou valor de MPV para a cidade de SJRP $\left(1,0 \mathrm{mg} \mathrm{L}^{-1}\right)$ inferior ao das cidades de Ribeirão Preto $\left(1,4 \mathrm{mg} \mathrm{L}^{-1}\right)$ e Campinas $\left(1,1 \mathrm{mg} \mathrm{L}^{-1}\right)$, mas superior ao das cidades de Santa Maria da Serra $\left(0,81 \mathrm{mg} \mathrm{L}^{-1}\right)$ e Figueira $\left(1,4 \mathrm{mg} \mathrm{L}^{-1}\right)$. Com relação ao $\mathrm{PO}_{4}{ }^{3-}$, TOC e NPOC não houve monitoramento nos referidos trabalhos (LARA et al., 2001). 


\section{Periódica Eletrânica}

\section{Fórum Ambiental}

Volume 14, Número 1, 2018

da Alta Paulista

ISSN 1980-0827

Tabela 2: Média ponderada pelo volume para as amostras de DT (MPV DT) e amostra de DU (MPV DU) para o período de safra e entressafra da cana-de-açúcar para a cidade de SJRP e média ponderada pelo volume para outras localidades.

\begin{tabular}{|c|c|c|c|c|c|c|c|c|c|}
\hline \multirow[b]{2}{*}{$\begin{array}{c}\text { Elementos } \\
\qquad\left(\mathrm{mg} \mathrm{L}^{-1}\right)\end{array}$} & \multicolumn{2}{|c|}{ MPV DT } & \multicolumn{2}{|c|}{ MPV DU } & \multicolumn{5}{|c|}{ Outras Localidades } \\
\hline & Safra & Entressafra & Safra & Entressafra & a & b & c & $d^{1}$ & $d^{2}$ \\
\hline $\mathrm{K}^{+}$ & 0,68 & 0,20 & 0,17 & 0,05 & 0,04 & - & 0,39 & 0,05 & 0,14 \\
\hline $\mathrm{NH}_{4}^{+}$ & 1,3 & 0,54 & 0,38 & 0,33 & - & 0,50 & 0,54 & 0,27 & 0,26 \\
\hline $\mathrm{SO}_{4}{ }^{2-}$ & 6,0 & 2,2 & 4,7 & 1,7 & - & 1,6 & 3,3 & 1,0 & 0,59 \\
\hline $\mathrm{PO}_{4}{ }^{3-}$ & 3,1 & 0,12 & 0,48 & 0,01 & - & - & - & - & - \\
\hline $\mathrm{NO}_{3}^{-}$ & 1,0 & 0,49 & 0,40 & 0,14 & - & 1,4 & 0,81 & 1,1 & 0,84 \\
\hline TOC & 7,1 & 5,4 & 1,4 & 1,8 & - & - & - & - & - \\
\hline NPOC & 6,0 & 5,2 & 1,2 & 1,8 & - & - & - & - & - \\
\hline
\end{tabular}

${ }^{\mathrm{a} C o e l h o ~ e t ~ a l ~(2008): ~ R i b e i r a ̃ o ~ P r e t o, ~ S a ̃ o ~ P a u l o, ~ B r a s i l ; ~}$

${ }^{\text {b} R o c h a ~ e t ~ a l ., ~(2003) ; ~ S a ̃ o ~ P a u l o, ~ S P ; ~}$

'Flues et al. (2002); Figueira, PR;

dLara et al (2001); ${ }^{1}$ Campinas; ${ }^{2}$ Santa Maria da Serra, SP.

- parâmetro não monitorado nos referidos trabalhos

Um estudo realizado por Campos et al. (2007) demonstrou que o carbono orgânico volátil (VOC), que é a diferença entre o carbono orgânico total e o carbono orgânico não purgável, presente nas águas de chuva da região da cidade de Ribeirão Preto se deve as condições de uso e produção de etanol na região, haja vista que em trabalho realizado no sudeste da Carolina do Norte (EUA), uma região que não apresenta as mesmas condições de plantio e consumo de etanol do Brasil, sendo que $86 \%$ das amostras apresentaram valores abaixo do LD do método $\left(<5 \mu \mathrm{M} \mathrm{L}^{-1}\right)$ para o a análise de carbono orgânico (AVERY JR. et al., 2009). Para a cidade de SJRP um considerável número de amostras, mais que 50\%, apresentou diferença variando de $7-70 \%$ para o VOC, podendo a 
presença deste composto estar relacionada com a utilização de álcool combustível em veículos automotores e/ou a queima incompleta da palha da cana-de-açúcar, como sugerido por Campos et al. (2007).

\section{Conclusão}

Foi observado que as maiores variações nas concentrações das amostras de DU para a cidade de SJRP foi na ordem $\mathrm{PO}_{4}{ }^{3-}>\mathrm{K}^{+}>\mathrm{NO}_{3}{ }^{-}>\mathrm{NPOC}>\mathrm{NH}_{4}{ }^{+}>\mathrm{C}$.E. $>\mathrm{SO}_{4}{ }^{2-} \sim \mathrm{TOC}>>\mathrm{pH}$, com as amostras variando desde o LD até 13,6; 5,26; 14,8; 29,9 e 5,6 $\mathrm{mg} \mathrm{L}^{-1}$ para $\mathrm{K}^{+}, \mathrm{NH}_{4}{ }^{+}, \mathrm{SO}_{4}{ }^{2-}, \mathrm{PO}_{4}{ }^{3-}$ e $\mathrm{NO}_{3}{ }^{-}$. Para o $\mathrm{pH} 18 \%$ das amostras apresentaram valor característico de chuva ácida, sendo que $76 \%$ dessas amostras foram quantificadas no período de safra da cana de açúcar.

Também foi observado que maior valor de pH $(7,28)$ ocorreu no mês de junho de 2010 , sendo este mês o subsequente de dois meses com as maiores quantidades de focos de queimada em conjunto com as menores pluviosidades mensais acumulada na região.

Já para as amostras de DT e DU a maior MPV ocorreu no período de safra da cana-de-açúcar para os elementos $\mathrm{K}^{+}, \mathrm{NH}_{4}{ }^{+}, \mathrm{SO}_{4}{ }^{2-}, \mathrm{PO}_{4}{ }^{3-}$ e $\mathrm{NO}_{3}{ }^{-}$, sendo que para $\mathrm{K}^{+}, \mathrm{NH}_{4}{ }^{+}$e $\mathrm{SO}_{4}{ }^{2-}$ os valores de MPV para a cidade de SJRP foram até 3,5 vezes superiores aos de cidades impactadas pelo cultivo da canade-açúcar no estado de SP como Ribeirão Preto, Campinas e Santa Maria da Serra e a cidade de Figueira (PR). Já o $\mathrm{NO}_{3}{ }^{-}$apresentou valor de MPV para a cidade de SJRP inferior ao das cidades de Ribeirão Preto e Campinas, mas superior ao das cidades de Santa Maria da Serra e Figueira.

Para TOC e NPOC foi observado que o maior valor de MPV ocorreu no período de safra da canade-açúcar para as amostras de DT e para as amostras de DU ocorreu no período de entressafra, sendo também que um considerável número de amostras, mais que $50 \%$, foram quantificáveis o VOC, o qual estar relacionada com a utilização de álcool combustível em veículos automotores e/ou a queima incompleta da palha da cana-de-açúcar.

Frente ao exposto é possível verificar que no período de colheita da cana-de-açúcar houve um aumento significativo de $\mathrm{K}^{+}, \mathrm{NH}_{4}{ }^{+}, \mathrm{SO}_{4}{ }^{2-}, \mathrm{PO}_{4}{ }^{3-}$ e $\mathrm{NO}_{3}{ }^{-}$, sugerindo com isso que essa prática agrícola possa estar impactando a atmosfera da região da cidade de SJRP com esses compostos. 


\title{
Agradecimentos
}

\author{
Os autores gostariam de agradecer a Fundação de Amparo a Pesquisa do Estado de São \\ Paulo (FAPESP) pelo apoio financeiro necessário a execução deste projeto (Processos 05/51242- \\ 8 e 09/03098-8).
}

\section{Referências}

ABNT. NBR 9898 - Preservação e técnicas de amostragem de afluente líquidos e corpos receptores - Procedimento, 1987.

APHA. Standar Methods for the Examination of Water and Wastwater. 21. ed. Washington, 2005.

AVERY, G. B. Jr., BROWN J. L. D., WILLEY J. D., KIEBER R. J. Assessment of rainwater volatile organic carbon in southeastern North Carolina, USA, Atmospheric Environment, v.43, p.2678-2681, 2009.

AYDIN I. AYDIN F., SAYDUT A., BAKIRDERE E. G., HAMANCI C. Hazardous metal geochemistry of sedimentary phosphate rock used for fertilizer (Mazıdag, SE Anatolia, Turkey). Microchemical Journal, v.96, p.247-251, 2010.

BAIRD, C. Environmental Chemistry. New York: W.H. Ferman and Company, 2002.

BAYRAKTAR, H.; TURALIOGLU, F. S. Composition of wet and bulk deposition in Erzurum, Turkey, Chemosphere, v.59, p.1537-1546, 2005.

CAFREY, P. F., ONDOV, J. M., ZUFALL, M. J., DAVIDSON, C. I. Determination of size dependent dry particle deposition velocities with multiple intrinsic elemental tracers. Environmental Science and Technology, v.32, p.1615-1622, 1998.

CAMPOS, M. L. A. M., NOGUEIRA, R. F. P., DAMETTO, P. R., FRANCISCO, J. G., COELHO, C. H. (2007). Dissolved organic carbon in rainwater: glassware decontamination and sample preservation and volatilite organic carbon, Atmospheric Environment, v.41, p.8924-8931.

CARDOSO, A.A., MACHADO, C.M.D., ALLEN, A.G. Atmospheric emission of reactive nitrogen during biofuel ethanol production, Environmental Science \& Technology, v.42, p.381-385, 2008.

CARVALHO JUNIOR, V.N. Deposição atmosférica e composição química da água de chuva, Revista Tecnológica, v.25, p.61-71.

CASTANHO, A. D. A. Determinação Quantitativa de Fontes de Material Particulado na atmosfera da cidade de São Paulo. Dissertação de Mestrado, Universidade de São Paulo, 1999. 
CHARLSON R. J., RODHE H. Factors controlling the acidity of natural Rainwater, Nature, v.295, p.683-685, 1982.

COELHO, C. H., FRANCISCO, J. G., NOGUEIRA, R. F. P., CAMPOS, M. L. A. M. Dissolved organic carbon in rainwater from areas heavily impacted by sugar cane burning, Atmospheric Environment, v.42, p.7115-7121, 2008.

CONKO, K. M., RICE, K. C., KENNEDY, M. M. Atmospheric wet deposition of trace elements to a suburban environment, Reston, Virginia, USA, Atmospheric Environment, v.38, p.4025-4033, 2004.

DER. Departamento Estadual de Estradas de Rodagem. (http://www.der.sp.gov.br/arquivos/documentos/frotaVeiculos2006.pdf, Acessado 18 Março 2011), 2006.

FELIX, E. P., CARDOSO, A. A. Amônia $\left(\mathrm{NH}_{3}\right)$ atmosférica: fontes, transformação, sorvedouros e métodos de análise, Química Nova, v.27, p.123-130, 2004.

FLUES, M., HAMA, P., LEMES, M. J. L., DANTAS, E. S. K., FORNARO, A. Evaluation of the rainwater acidity of a rural region due to a coal-fire power plant in Brazil, Atmosheric Environment, v.36, p.2397-2404, 2002.

HU, G.-P., BALASUBRAMANIAN, R. Wet deposition of trace metals in Singapore, Water, Air and Soil Pollution, 144, 285300, 2003.

INPE . Instituto Nacional de Pesquisas Espaciais (www.http://www.dpi.inpe.br/proarco/bdqueimadas/. Acessado em 05 Março 2011), 2011.

KROM, M. D. Spectrophotometric Determination of Ammonia:A Study of a Modified Berthelot Reaction Using Salicylate and Dichloroisocyanurato, The Analyst, v.105, p.305-316, 1980.

LARA, L. B. L. S., ARTAXO, P., MARTINelLI, L. A., VICTORIA, R. L., CAMARgO P. B., KRUSCHE A. ET AL. Chemical composition of rainwater ans anthropogenic influences in the Piracicaba Rver Basin, Southeast Brazil, Atmospheric Environment, v.35, p.4937-4945, 2001.

LARA, L. L., ARTAXO, P., MARTINELLI, L. A., CAMARGO, P. B., VICTORIA, R. L., FERRAZ, E. S. B. Properties of aerosols from sugar-cane burning emissions in Southeastern Brazil, Atmospheric Environment, v.39, p.4627-4637, 2005.

LIVINGSTONE, C., RIEGER, P., WINER, A. Ammonia emission from a representative in-use fleet of light and medium-duty vehicles in the California South Coast Air Basin, Atmospheric Environment, v.43, p.3326-3333, 2009.

MACHADO, C. M. D., CARDOSO, A. A., ALLEN, A. G. Atmospheric emission of reactive nitrogem during biofuel ethanol production, Environmental Science \& Technology, v.42, p.381-385, 2008.

MARTINS, C. R., ANDRADE, J. B. Química atmosférica do enxofre (IV): emissões, reações em fase aquosa e impacto ambiental, Química Nova, v.25, p.259-272, 2002. 


\section{Periódica Eletrânica}

\section{Fórum Ambiental}

MIGLIAVACCA, D. M., TEIXEIRA, E. C., GERVASONIC, F., CONCEIÇÃO, R. V., RODRIGUEZ, M. T. R. Characterization of wet precipitation by Xray diffraction (XRD) and scanning electron microscopy (SEM) in the metropolitan area of Porto Alegre, Brazil, Journal of Hazardous Materials, v.171, p.230-240, 2009.

MIGON, C., SANDRONI, V. Phosphorus in rainwater: Partioning inputs and impact on the surface coastal ocean, Limnology and Oceanography, v.44, p.1160-1165, 1999.

MAPA . Ministério da Agricultura, Pecuária e Abastecimento- Brasil. Balanço nacional de cana-de açúcar e agroenergia. Secretaria de Produção e Agroenergia- Brasília, MAPA/SPAE, 2007.

NICHOLSON K. W. A Review of particle ressuspension, Atmospheric Environment, v.22, p.2639-2651, 1988.

PIRES, D. O. Inventário de emissões atmosféricas de fontes estacionárias e sua contribuição para a poluição do ar na região metropolitana do Rio de Janeiro. Dissertação de Mestrado, Universidade Federal do Rio de Janeiro, 2005.

ROCHA, F. R., DA SILVA, J. A. F., LAGO, C. L., FORNARO, A., GUTZ, I. G. R. Wet deposition and related atmospheric chemistry in the São Paulo metropolis, Brazil: Part 1. Major inorganic ions in rainwater as evaluated by caillary electrophoresis with contacless condutivity detection, Atmospheric Environment, v.37, p.105-115, 2003.

ROCHA, J. C., ROSA, A. H., CARDOSO, A. A. Introdução à Química Ambiental. 2. ed. Porto Alegre: Bookman, 2009.

RYU S. Y., KWON B. G., KIM Y. J., CHUM K. J. Characteristics of biomass burning aerosol and its impact on regional air quality in the summer of 2003 at Gwangju, Korea, Atmospheric Research, v.84, p.362-373, 2007.

SILVA, M. P. R. Modelagem numérica dos processos de remoção úmida de poluentes atmosféricos: estudo de caso para região amazônica (Rondônia). Dissertação de Mestrado, Universidade de São Paulo, 2006.

SOUZA, P. A.; MELLO, W. Z., MALDONADO, J., EVANGELISTA, H. Composição química da chuva e aporte atmosférico na Ilha Grande, RJ, Química Nova, v.29, p.471-476, 2006.

STERNBECK J., SJÖDIN A., ANDRÉASSON K. Metal emissions from road traffic and the influence of resuspension-results from two tunnel studies, Atmospheric Environment, v.36, p.4735-4744, 2002.

TSUKUDA, S. SUGIYAMA, M. HARITA, Y., NISHIMURA, K. Atmospheric bulk deposition of soluble phosphorus in Ashiu Experimental Forest, Cental Japan: sources apportionment and sample contamination problem, Atmospheric Environment, v.39, p.823-836.

VASCONCELOS, P. C., BALASUBRAMANIAN, R., BRUNS, R. E., SANCHEZ-CCOYLLO O., ANDRADE, M. F., FLUES, M. Watersoluble ions and trace metal in airborn particles over urban areas of the state of São Paulo: influences of local sourcers and long range transport, Water, Air \& Soil Pollution, v.186, p.63-76, 2007. 